\title{
An Investigation of the Impact of Aerodynamic Model Fidelity on Close-In Combat Effectiveness Prediction in Piloted Simulation
}

\author{
T. Ray Persing*, Christine A. Bellish $\dagger$ \\ National Air and Space Intelligence Center, 4180 Watson Way, Wright-Patterson Air Force Base, Ohio $45433-5648$ \\ Jay Brandon $\ddagger$ P. Sean Kenney§ \\ NASA Langley Research Center, Hampton, VA 23681-2199

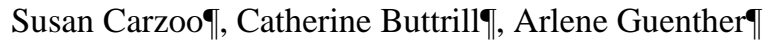 \\ Unisys Corporation, 20 Research Drive, Hampton, VA 23666
}

\begin{abstract}
Several aircraft airframe modeling approaches are currently being used in the DoD community for acquisition, threat evaluation, training, and other purposes. To date there has been no clear empirical study of the impact of airframe simulation fidelity on piloted real-time aircraft simulation study results, or when use of a particular level of fidelity is indicated. This paper documents a series of piloted simulation studies using three different levels of airframe model fidelity. This study was conducted using the NASA Langley Differential Maneuvering Simulator. Evaluations were conducted with three pilots for scenarios requiring extensive maneuvering of the airplanes during air combat. In many cases, a low-fidelity modified point-mass model may be sufficient to evaluate the combat effectiveness of the aircraft. However, in cases where high angle-of-attack flying qualities and aerodynamic performance are a factor or when precision tracking ability of the aircraft must be represented, use of high-fidelity models is indicated.
\end{abstract}

DMS - Differential Maneuvering Simulator

\section{Nomenclature}

NASIC - National Air and Space Intelligence Center

NASA-LaRC - National Aeronautics and Space Administration Langley Research Center

$6 D O F$ - Six degree-of-freedom

5DOF - Five degree-of-freedom

HOBS - High off-boresight

AFRL - Air Force Research Laboratory

$B V R$ - Beyond Visual Range

WVR - Within Visual Range

CIC - Close-in Combat

$V c-$ Closing velocity

AGL - Above Ground Level

GEM3 - Fully developed 6-DOF aircraft model

SCaRE - Limited-scope 6-DOF aircraft model

Hercules - Low-fidelity modified point mass aircraft model

HMCS - Helmet mounted cueing system

HUD - Head Up Display

* - Principal Intelligence Analyst, NASIC/AENE, AIAA Member

$\dagger$ - Combat Effectiveness Analyst, NASIC/AENE

$\ddagger$ - Aerospace Engineer, Flight Dynamics Branch, AIAA Associate Fellow

$\S$ - Aerospace Engineer, Flight Simulation and Software Branch, AIAA Member

If - Software Engineer, U.S. Federal Government Group 


\section{BACKGROUND}

The instinctive reaction to a modeling task is frequently to use the highest fidelity model available. The National Air and Space Intelligence Center (NASIC) has had many instances where a client has requested a six degree-offreedom (6DOF) aircraft flight dynamics model for an application that does not immediately appear to have a requirement for high fidelity airframe modeling.

Developing high-fidelity aircraft handling qualities models can incur considerable expense. For an analytic level 3DOF aircraft model with second-order responses appended to provide simplistic rotational response, the cost is negligible - limited to the pay of the analyst. For a 6DOF model valid through the trimmed flight envelope, developed through a combination of analysis and small-scale wind tunnel testing, the cost is typically on the order of $\$ 400,000$ per model. For a post-stall capable model, with a aerodynamic database developed for the entire possible range of motion, with dynamic derivatives determined through dynamic testing of large, and sometimes dynamically-scaled models, cost is measured in millions of dollars.

It is thus pragmatic to develop the aircraft model only to the level of detail that is required for a particular simulation application. As noted in the previous paragraph, the cost of development of the simulation models increases exponentially as fidelity increases. Unfortunately, the benefits of increased fidelity have not been quantified. Responsible use of resources, and assurance of adequate fidelity to insure valid conclusions highlight the need for development of guidelines to enable rational decision making when developing simulation models for a specific task.

A previous study ${ }^{1}$ conducted by AFRL and NASIC compared 3 levels of aircraft fidelity in beyond-visual-range (BVR) situations. This study compared a full 6DOF representation of the airframe aerodynamics, a 6DOF equivalent system representation of the airplane which represents the airplane dynamics with transfer functions described by frequencies and damping ratios for the classic airplane response modes, and a 5DOF equivalent system which deleted the yaw degree of freedom. The results of these studies showed no effect of predicted airplane combat effectiveness in the BVR arena due to the model. An unexpected result was that simulator visual system characteristics did have an impact of predicted effectiveness, even in the BVR (limited maneuvering) engagements.

To extend the study of airframe fidelity effects, the National Air and Space Intelligence Center (NASIC) and the National Aeronautics and Space Administration's Langley Research Center (NASA-LaRC) cooperatively developed a research project to determine the impact of simulation fidelity on piloted real-time aircraft simulation study results. This study expanded the BVR results into the within-visual-range (WVR) arena, looking at highly dynamic close-in combat (CIC) scenarios.

This study was jointly funded by The Department of Defense, Director Operational Test and Evaluation, Threat Systems Office, NASA-LaRC, and NASIC.

\section{STUDY DESIGN}

The key aspect of the study was to provide insight into the level of fidelity and "rigor" of the aerodynamic math models of the aircraft airframe model required to obtain representative results for close-in air combat (CIC) evaluations. To address this issue, three mathematical models of the same airplane configuration were developed at different aerodynamic fidelity levels. The lowest fidelity aerodynamic model consisted of a point-mass with specified performance and rate capabilities - i.e. no explicit aerodynamic model at all. The medium fidelity aerodynamic model was derived from a series of wind tunnel tests at a small low-speed wind tunnel facility using inexpensive epoxy wind-tunnel models developed with stereo-lithography techniques. The highest fidelity aerodynamic model was developed using a large-scale wind tunnel model - similar to that used in all recent fighter airplane development programs - which included testing in a large subsonic wind tunnel for static and dynamic aerodynamic stability characteristics. 
To separate effects due to modeling fidelity from other potential variables, the three versions of the same airplane were adjusted to give comparable levels of performance - at least over the normal flight envelope - so that standard comparisons of specific energy capabilities, turn rates, energy addition capabilities, and sustained g capabilities were identical. Additionally, systems required for the CIC tasks, such as radar, missiles, guns, and displays were identically modeled for each of the airplane configurations. The airplane simulated is similar to current $4^{\text {th }}$-generation fighters with a sea-level thrust-to-weight ratio of approximately 1 , maneuver g-capability of 9 g's, and a corner speed of approximately 370 knots calibrated airspeed (KCAS).

Piloted simulation studies were conducted using the NASA Langley Differential Maneuvering Simulator (DMS). The DMS is a facility with two 40-foot diameter spheres with a generic cockpit in each. Each sphere has the adversary airplane projected onto the scene to allow for cooperative or combat maneuvering. Piloted evaluations were conducted with three pilots for scenarios requiring extensive maneuvering of the airplanes during air combat. Each airplane was equipped with identical weapon systems consisting of radar, a gun with lead-computing sight, and short-range IR missiles. For some engagements, the missiles were all-aspect capable, and the pilots were given a helmetmounted sight capability.

Two aircraft initial condition setups allowed for examination of differences in both a traditional turning fight, and a low-speed, high angle-of-attack 'scissors' fight. These scenarios are illustrated in Figures 1 and 2.

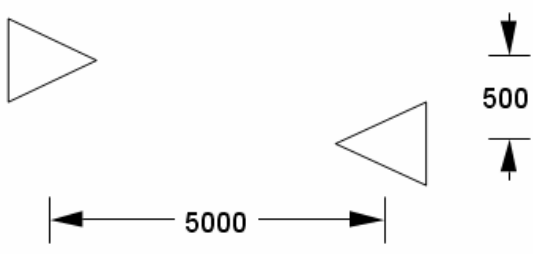

- Slightly above Vc at merge (425 KCAS)

- 2-Circle initially

- No maneuvering till "fight's on" tone

Figure 1 - High-aspect test initial condition

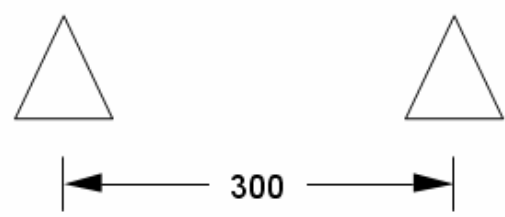

- 150 KCAS initial speed

Figure 2 - Scissors low-speed test initial condition

In the first case, referred to as the 'high-aspect' setup, the aircraft are initially positioned at approximately 1 mile apart, nose-to-nose, with a $500 \mathrm{ft}$ lateral offset. The initial velocity was set to slightly above the aircraft corner speed, and pilots were constrained to an initial two-circle tactic. Additionally, no maneuvering was permitted until the aircraft had passed each other. At this point, the pilots aggressively maneuvered the aircraft to obtain the first shot opportunity. This provided evaluation points for traditional turning combat.

The second scenario was designed to force the pilots into a scissors fight in the low-speed, high-alpha regime representing a condition in which two equally matched aircraft had fought to a point of low specific energy.

All cases were started at an altitude of 15,000 ft AGL. A 5,000 ft hard deck was enforced - any aircraft descending below 5,000 ft was declared a 'rocks kill.' Finally, the length of an engagement was capped at 2 minutes.

\section{MODEL DESCRIPTIONS}

\section{A. Aerodynamic Models}

Traditional airframe aerodynamics models consist of detailed mathematical representation of the forces and moments acting on the airplane over a wide range of flight conditions and control inputs. Typical aerodynamic 
models are implemented as truncated Taylor series with first-order and sometimes higher order terms describing the aerodynamic characteristics due to important parameters such as angle of attack $(\alpha)$, angle of sideslip ( $\beta$ ), Mach number, rotational rates, control surface deflections, and other parameters ${ }^{2}$. Although computational fluid dynamics capabilities have improved dramatically over the past several years, accurate prediction of stability and control aerodynamic parameters - especially at high angular rates and any flight condition with large areas of separated flow - requires experimentally-derived data. Large high-fidelity wind tunnel databases can be very costly to develop.

Three levels of fidelity of airframe aerodynamic modeling were evaluated during the study. The high fidelity model (GEM3) was developed using a conventional set of wind tunnel tests with a large model tested statically and dynamically in a large subsonic wind tunnel. This large data set resulted in a very nonlinear, extensive set of data tables which were used as the simulation database. Experimental data were available for a very large range of $\alpha$ ($\left.180^{\circ}<\alpha<180^{\circ}\right)$ and $\beta(-90<\beta<90)$. Mach effects were estimated using analytic and empirical techniques.

Flight controls were developed based on current $4^{\text {th }}$-generation fighter flight control system designs. Development cost for the GEM3 model was approximately \$1.7 million.

The medium-fidelity aerodynamic model (SCaRE) was developed using a smaller, lower fidelity wind tunnel model constructed of relatively inexpensive epoxy material using a stereo-lithography model construction technique. The model was then statically tested in a relatively small subsonic wind tunnel facility. The range of experimental data was at low subsonic speeds, with angle of attack ranges from -10 to approximately 35 degrees; sideslip tests were run at $0,-5$, and +5 degrees. Mach effects and dynamic stability characteristics were estimated using analytic and empirical techniques.

The equations used in the SCaRE model aerodynamics are roughly equivalent to the ones used in the higher fidelity model, however, because of the methods used to generate it, the quality of the data used in the SCaRE model is significantly lower than in the previously discussed GEM3 model. Additionally, flight controls were developed using a perfect knowledge of the aerodynamics. This allowed for tailoring of the responses of the airplane to ensure Level I flying qualities in all expected flight conditions. The level of control of such things as coordination of rolls is significantly greater than current actual flight control systems are capable of. One artifact noted in this model was the existence of an unrecoverable spin-like departure which occasionally resulted from extensive aggressive maneuvering. This departure mode was not thought to be representative of the test aircraft configuration. Total development cost for the SCaRE model was approximately $\$ 400 \mathrm{~K}$.

The low-fidelity aerodynamic model (Hercules) was developed by use of analytic and empirical methods to estimate the lift generation capabilities of the airplane, and drag characteristics to result in the desired energy capabilities. The rest of the model for the low-fidelity case did not explicitly represent aerodynamic properties at all. Instead, the airplane dynamics were represented by transfer functions designed to give Level I flying qualities throughout the flight envelope. Cost for developing the Hercules model was not specifically tabulated, but development of the model occurs with approximately $1 / 4$ - man-year effort using existing tools and procedures.

\section{B. Airframe Model Verification}

As noted, one of the objectives was to evaluate the impact of analytic rigor in the model development, and thereby the effects of spending additional resources on the airframe models. However, in order to isolate the effects of flight dynamics modeling from the aircraft's kinematic performance, the researchers felt it important to 'tune' the kinematic performance of the different models.

The Hercules model was used as the kinematic baseline for this study, because it represents the methods used for development of data used in US Department of Defense weapons handbooks. The GEM and SCaRE models used the same engine models for thrust and fuel flow vs. power level angle (PLA) as the Hercules model. In order to match the drag characteristics of the Hercules model, the GEM and SCaRE models had an additional drag correction factor added into the $C_{D}$ Taylor series expansion so that the drag as a function of lift were identical for all three models. This provides for identical specific power calculations for all three aircraft across the board. These corrections were verified in the simulator by checking $1 \mathrm{G}$ level acceleration characteristics and sustained turn performance. 


\section{Drag Polar Comparison \\ 1-g trims at $10000 \mathrm{ft}$}

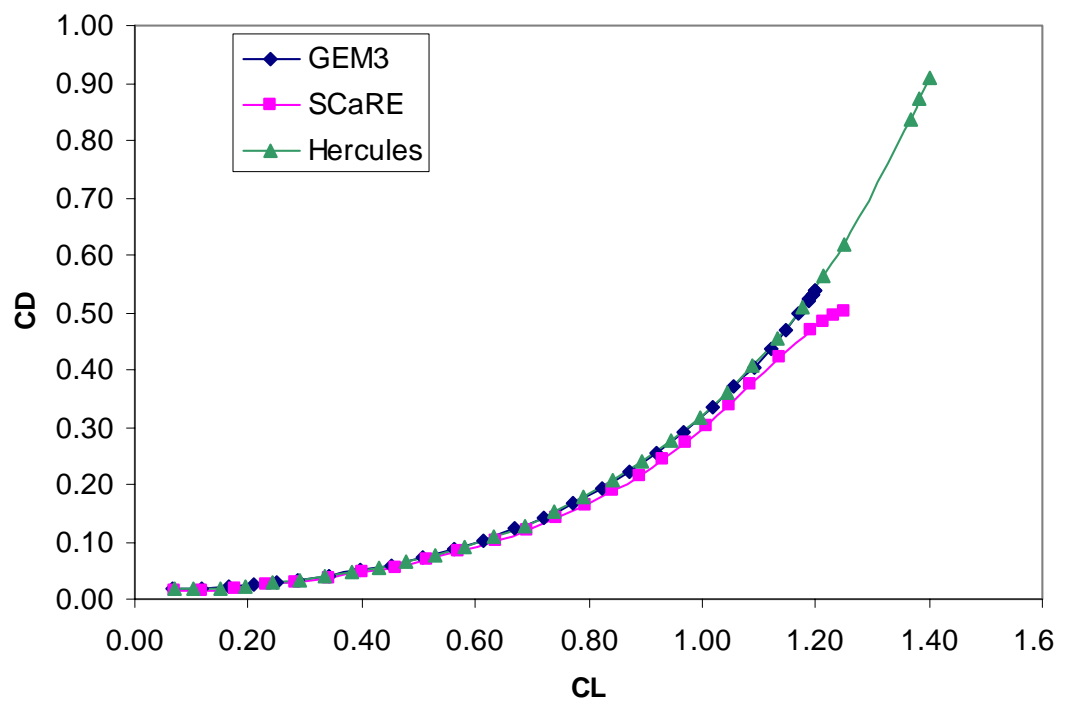

Figure 3 - Drag polar comparison, GEM3 vs. SCaRE vs. Hercules

\section{Trimmed Lift \\ 1-g trims at $10000 \mathrm{ft}$}

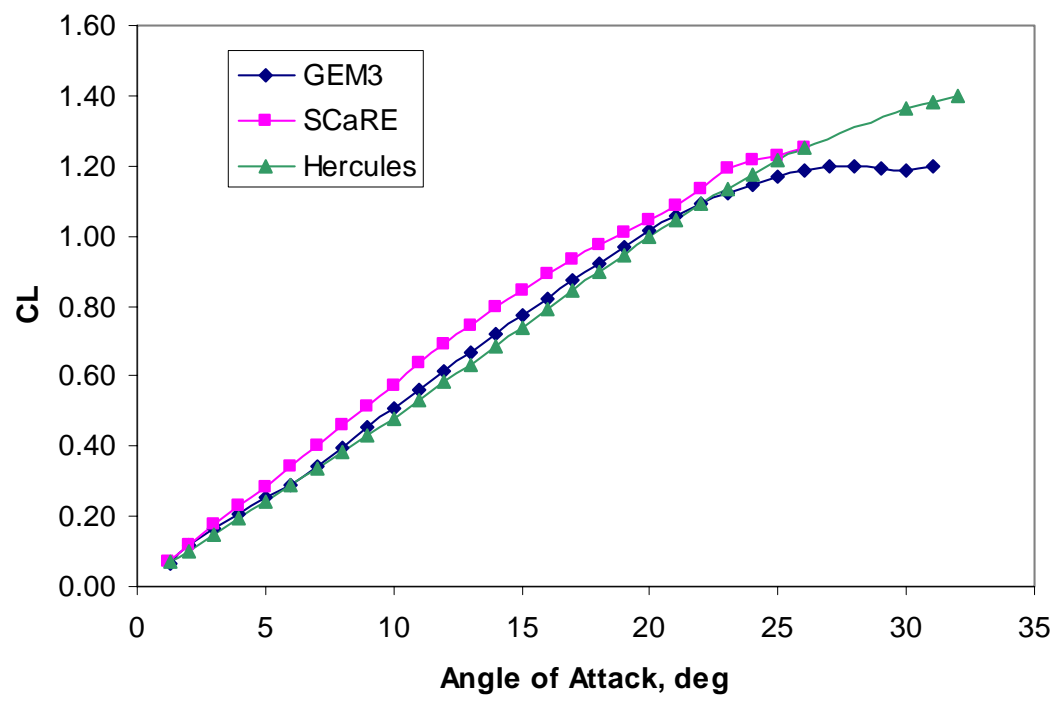

Figure 4 - 1G Trimmed lift, GEM3 vs. SCaRE vs. Hercules

The only significant area of difference in what is traditionally a classical performance area is the high- $\alpha$ lift characteristics of the vehicle. The Hercules methodology does not do an explicit trim of the vehicle using moment balances, but rather assesses a maximum attainable lift and corresponding angle of attack based upon preliminary design analytic methods. This area was felt to be an area that was inextricably tied to the flight dynamics analysis rigor, and therefore trimmed lift capability was left independent of basic kinematic tuning. In most areas, the lift and drag curves matched relatively well. However, at higher angles of attack, differences manifested that were to eventually have an effect on the test results. Figure 3 shows the trimmed drag polars for the three models of the aircraft which show almost identical characteristics - at least until very high lift coefficient values. Figure 4 shows the lift coefficient as a function of $\alpha$. The data show small differences in the lift-curves, with the largest difference being the higher trimmed lift obtained with the Hercules model compared to the others. Each of the airplane configurations could trim up to slightly over $30^{\circ} \alpha$. No modifications of the aerodynamic models were made to attempt to align lift vs. $\alpha$ characteristics.

\section{Weapon and Avionics Models}

The weapons and avionics models were identical for each aerodynamic model. An airborne intercept radar was modeled (Figure 5). The radar was in auto-acquisition mode. Lockon to a target required 2 seconds. The radar antenna had a $\pm 60^{\circ}$ gimbal limit. With missiles selected, the radar reverted to a $\pm 20^{\circ}$ by $\pm 20^{\circ}$ autoacq volume. With guns selected, the radar search volume was set to $\pm 30^{\circ}$ azimuth and $\pm 10^{\circ}$ elevation.

The radar model provides a generic representation of the performance of airborne radar. The model is made up of many component models that together are used to represent the operation of a modern aircraft radar system. The radar model allows for multiple antennas and features a multi-mode capability that constrains the radar to a set of allowed operations (e.g. acquire, scan, track) per mode. Currently the radar model has only been tested/used in a single-target-track mode. In operation, the radar model reviews all of the positions and signatures of targets 


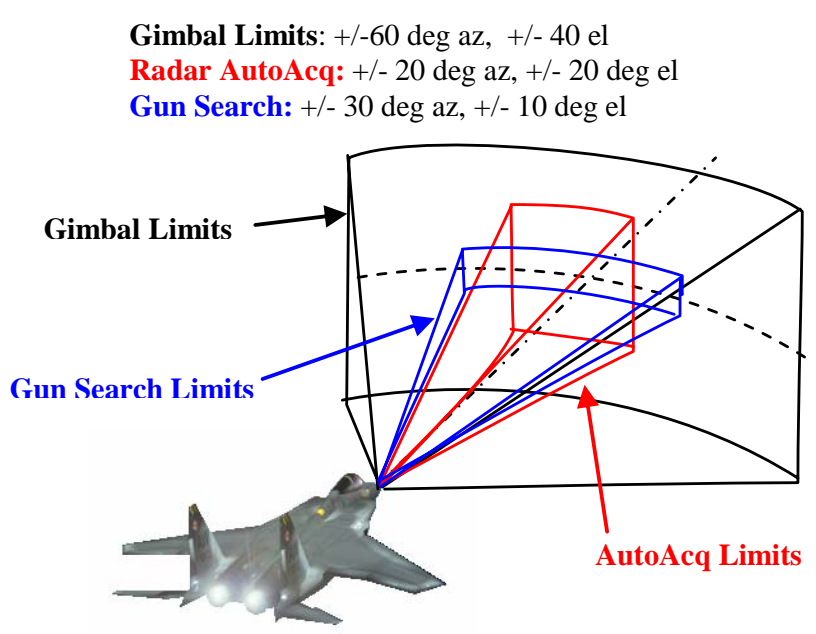

Figure 5- Radar field of view and field of regard limits available for detection and uses the radar range equation to compute a signal to noise ratio for targets detectable by its antenna(s). The radar model relies on parameter sets to configure the performance of the radar. Users can configure a wide variety of parameters such as the signal-tonoise threshold, dwell time, scan patterns, detection wavelengths, transmitter loss, receiver noise, and receiver loss.

Both aircraft were equipped with a rudimentary radar warning receiver (RWR) model. The RWR is constructed from the same components as the radar model. Essentially the RWR is a radar model operating only in a passive mode. Users can configure performance parameters just like the radar model. Typically the RWR has many antennas whereas the radar on fighter aircraft usually has one primary antenna.

Two missile models were used during the tests. A rear-aspect missile, similar to an AIM-9P was simulated which was effective against targets at $\pm 45^{\circ}$ aspect. The missile IR seeker limits were $\pm 45^{\circ}$. A fusing delay of 2 seconds was modeled. The other missile model was an all-aspect, high-off-boresight capable missile (HOBS) with $360^{\circ}$ aspect effectiveness, and an IR seeker limit of $\pm 90^{\circ}$. The all-aspect missile seeker was slaved to a helmet-mounted cueing system (HMCS) which slewed both the airplane radar antenna, and the missile seeker. Fusing delay was also set to 2 seconds.

The missile model was designed to provide a generic missile capability that could be configured to represent many different types of missiles. The model is a 6 degree-of-freedom fly-out model. The primary components of the model are the aerodynamics, flight control system, and propulsion system. All parameters of these sub models may be modified to alter the performance of the missile. The default parameter set models an AIM-9 Sidewinder. The control system uses the classic proportional navigation guidance law to intercept the target tracked by its seeker. Proximity and impact fuse models are used to determine whether or not the missile destroys its target.

The infrared seeker model design is similar to that of the radar model. The seeker is intended to provide a generic representation of the performance of an infrared seeker. The model uses the positions and signatures of targets available for detection and computes a detection range using signal to noise ratio using atmospheric transmission properties and performance parameters. The target with the largest detection range that is within the seeker's field of view is tracked provided it is within the minimum detection range.

\section{Simulation Facility}

The tests were conducted in the NASA Langley Differential Maneuvering Simulator (DMS). The DMS hardware consists of two 40 -foot diameter spheres with generic cockpits at the center of each sphere (Figure 6). The cockpit out-the-canopy display is computer generated imagery of the local area (Figure 7). The cockpit panel includes 3 multifunction displays which are programmable to represent systems of interest during research in the DMS. For the current study, an engine display, head-up display (HUD) repeater, and a radar warning display were selected. The cockpit also used a HUD for the basic flight data and weapon

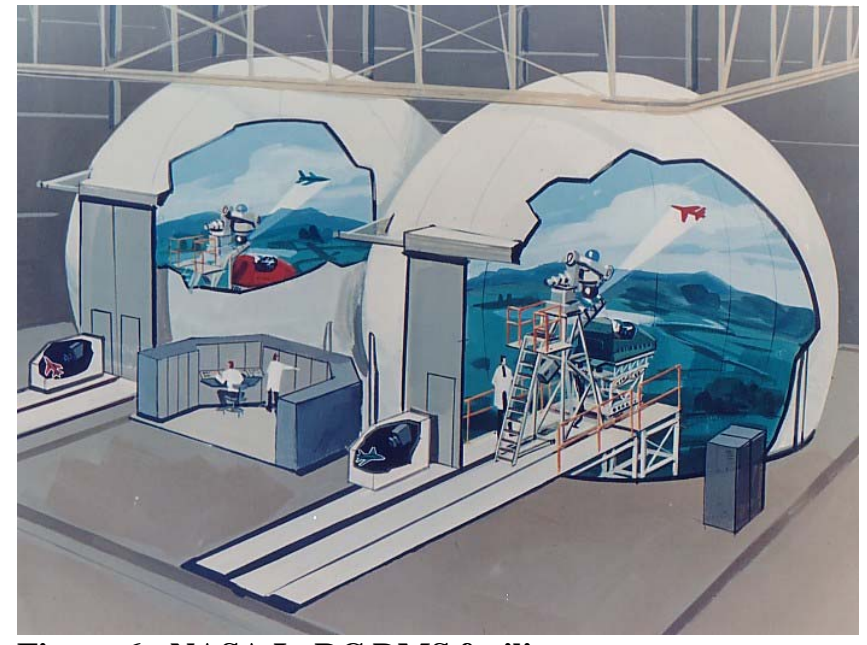

Figure 6 - NASA LaRC DMS facility 


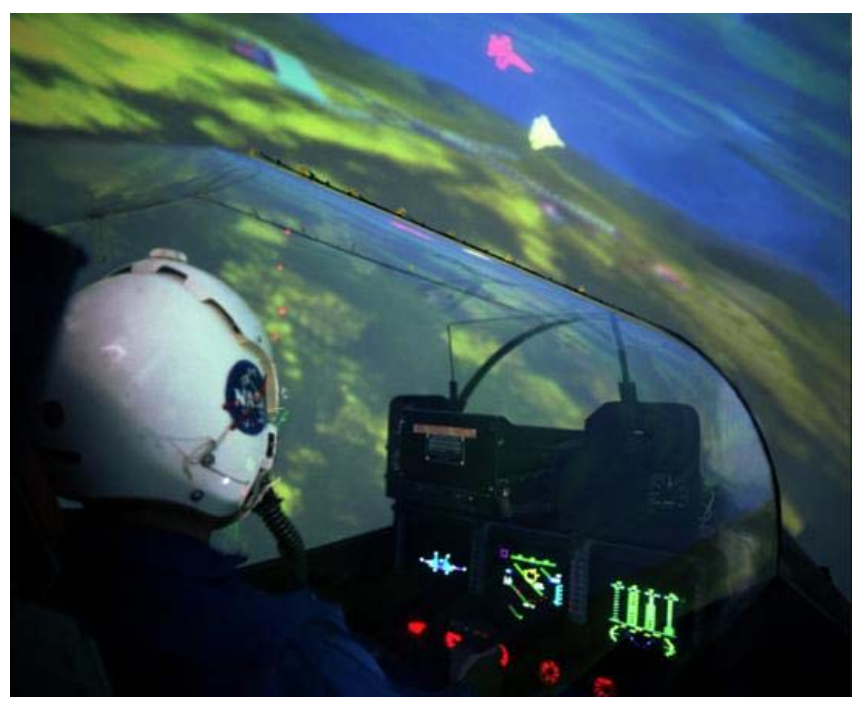

Figure 7 - Interior, NASA LaRC DMS sphere

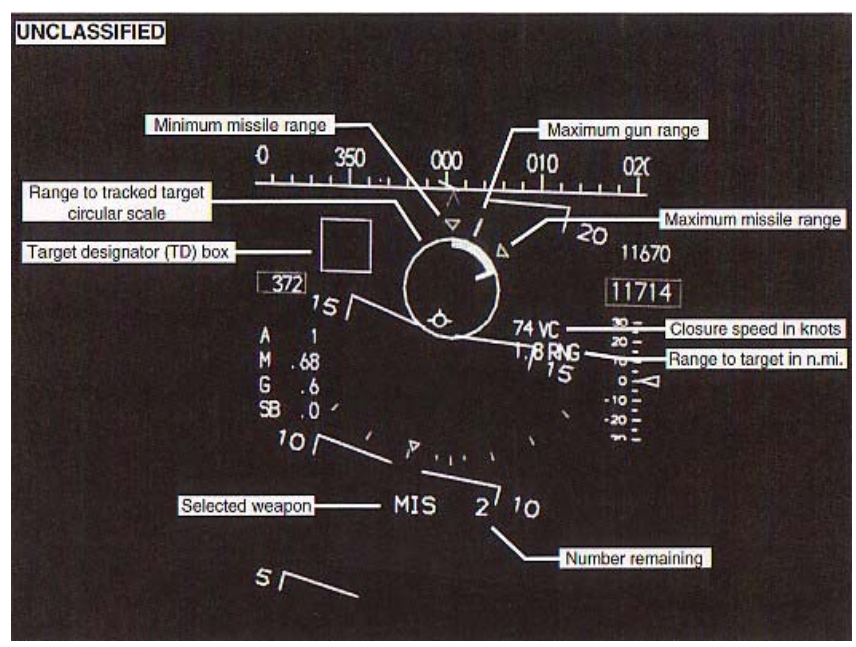

Figure 8 - DMS HUD representation system information (Figure 8). When using the all-aspect weapons, a helmet-mounted cueing system (HMCS) was employed to slew the radar antenna and missile seekers for target acquisition. The HMCS was a magnetic head tracking system attached to a laser pointer which shown on the inside of the DMS sphere to act as a sighting reticle. The cockpits are equipped with programmable force-feel systems for the center sticks and rudder pedals. An identical set of characteristics for the force-feel systems was used for each of the models tested.

The simulation mathematical models were hosted in LaSRS-C $++^{3}$ and included the airframe models, fly-out missile models, and the avionics systems.

\section{TEST TECHNIQUES}

Three pilots with Air Force or Navy tactical jet backgrounds participated in the combat effectiveness evaluation phase of the study. Four basic scenarios were evaluated with all perturbations of aircraft and pilot combinations. For each scenario, each airplane had identical weapon systems and initial energy states. One half of the test matrix is shown in Table 1 , indicating the scenarios flown with conventional weapons. The matrix was repeated with high-offboresight missiles and the HMCS.

The first scenario was an engagement beginning just above corner speed at a neutral merge. This was presented earlier as the 'high-aspect' setup. The weapons for this scenario consisted of tailaspect missiles, a 30-mm cannon, and a radar. The missile's performance and seeker/tracker capabilities were similar to an AIM-9P, though no

countermeasures or ground clutter, etc. were modeled.

The second scenario, presented earlier as the 'Scissors Fight', consisted of a low-speed fight - initially close abeam. This was designed to evolve into a slow-speed scissors fight. These two scenarios were picked to force extensive maneuvering and were somewhat artificial tasks in light of todays all-aspect, high-off boresight missile capabilities. The third and fourth scenarios comprised the same initial conditions as the previous ones, but included the high-offboresight and all-aspect missile capabilities and used the HMCS to target the missile seeker.

Each engagement was limited to 2 minutes, and the engagements were repeated at least 5 times with each pilot/configuration pairing. A round-robin series of testing was conducted pairing all combinations of pilots, airplane models, air combat task, and weapons to reduce effects of pilot performance variations. Tests were conducted "blind" with the pilots not being advised which airplane model they were flying. This procedure enabled a measure of statistical significance of results. Recorded parameters included kills, first shot opportunities, pilot comments, and time-history recordings of the engagements.

Before the data were obtained with the subject airplane models, a baselining phase of the tests was conducted in the DMS using the subject tasks, but with another airplane model that each pilot flew against one another. All 
combinations of pilots and tasks were conducted to determine uncertainty levels to be expected in the study results, and to provide training for the pilots in the DMS environment. Following the initial baselining phase, pilots were rotated in the various airplane configurations without explicitly knowing which model they were flying, or were flying against. The study was conducted over a three-day period with 424 simulated combat engagements being recorded for the study.

Raw data after each engagement was tabulated - consisting primarily of number of missiles and/or bullets fired, and status (dead or alive). Data were recorded for each of the engagements used for research to allow for post-test analysis of the results in more detail.

\section{RESULTS}

Two sets of engagement results were obtained from this study. The first was kill ratio information. The kill ratios were available immediately after a series of engagements, and were collected and analyzed as functions of pilots, configurations, weapons, and scenarios. The second set of results was more detailed engagement analysis conducted after the tests were completed. These analyses were conducted by reviewing the time history data from all the runs, and compiling more complete information about what happened during the study. Major parameters of interest in the engagement analysis were first shot opportunities and time at advantage relative to the other airplane in the engagement. This data could be used to reduce pilot weapon employment proficiency issues, missile model uncertainties, and other undesirable sources of variation in the kill ratio results.

\section{A. Kill Ratio Results}

The most straight-forward data from a series of combat engagements is the cataloging of final results: kill ratios. All ratio data will be presented as the data for the higher fidelity airframe model divided by the data for the lower fidelity airframe model. A green line is indicated on the figures at the kill ratio of 1 . For values below the green line, the higher fidelity model of the pair indicated on the figure was seen to have a disadvantage. Likewise, for values above the green line, the higher fidelity model exhibited an advantage over the lower.

These results are shown in Figures 9 through 11. The data in Figures 9 and 11 represent both the conventional weapons case (bar on the left of each model pairing) and the HOBS case (bar on the right of each model pairing), while Figure 10 addresses only high off-boresight weapons in the high-aspect scenario (removing results from gun or "rock" kills).

The kill ratios show a mixed result for the high-aspect cases. For conventional weapons in the high-aspect setups, the GEM3 showed a disadvantage relative to the less sophisticated SCaRE model by about a 2 to 1 ratio. The GEM3 was then slightly better than the Hercules model. The SCaRE model showed slightly lower effectiveness compared to the Hercules model. These results do not provide a consistent answer for the impact of model fidelity in terms of raw exchange ratios. For high-off-boresight weapons, again an inconsistent set of trends emerge from the 
raw data, though in this case it shows that the high-fidelity model is consistently at a disadvantage relative to the other models, but the medium-fidelity model (SCaRE) shows an advantage relative to the low-fidelity model (Hercules).

One test artifact that was noted during the data collection was that the pilots often employed the gun with "face shots" as the airplanes reached each merge during the high-aspect scenarios. The high aspect tasks were intended to be a missile weapon task, and use of the head-on "face shots" at the merges certainly tended to cloud the results of maneuvering the airplane for rear-aspect shots. For high-off-boresight weapons, the expected result was a turn from across the turning circle at a large off-boresight angle. When the data were distilled to include only the high-off-boresight missile kills for the high aspect scenario, the three models were much more even. Figure 10 shows the results, which as expected, are approximately equal. As the weapon system becomes more capable, the launch platform becomes less of a dominating factor.

In the low-speed cases, the results seen in Figure 11 show that while the GEM and SCaRE models were fairly evenly matched in the conventional weapons case, in the high off-boresight cases, the GEM model had a significant advantage - which seems to be counter-intuitive, as the introduction of HOBS weapons should tend to equalize the capabilities, not exacerbate differences.

Despite this, an obvious trend evident in Figure 11 is that the Hercules model had a significant advantage over the two more sophisticated models in the low-speed scissors fights. This is directly attributable to the Hercules model having extremely stable nose pointing capability in comparison to the other models, and in higher maximum trimmed lift capability which enabled the Hercules model to fly slower and maneuver behind his opponents. All pilots commented on the Hercules model's rock-steady tracking ability, regardless of the maneuvering situation - an unrealistic capability for an aircraft.

Pilot proficiency was also a factor, despite attempts to reduce it as a variable through the round-robin testing. There were clear pilot advantages in various scenarios, as seen in Table 2. Additionally, weapons system employment sometimes became an issue. The shoot cue was

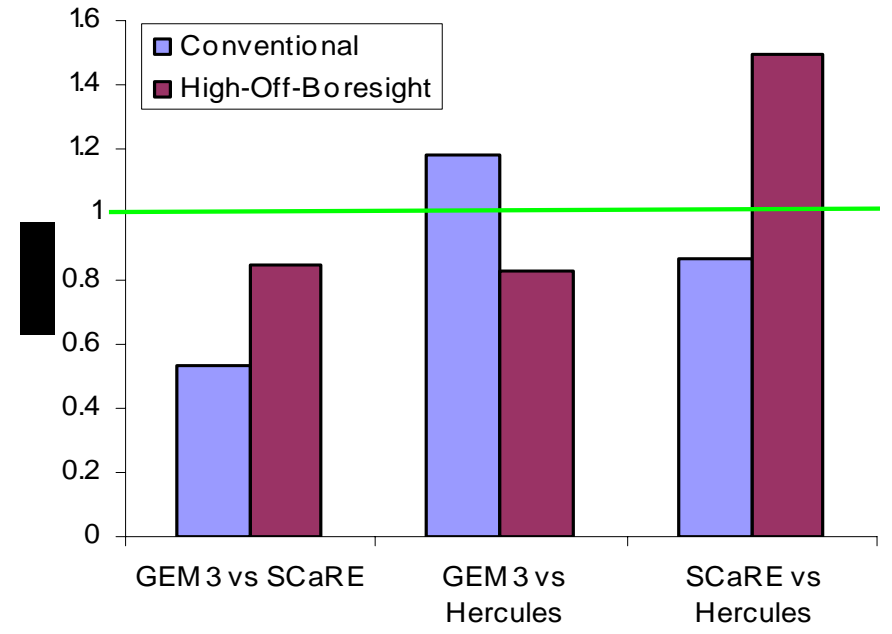

Figure 9 - High aspect scenario kill ratio results

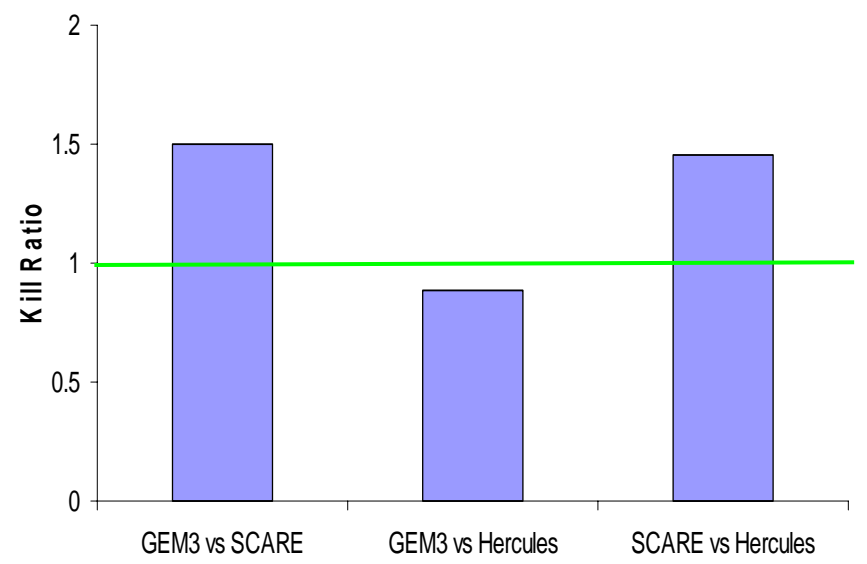

Figure 10 - High aspect scenario with HOBS missiles Only

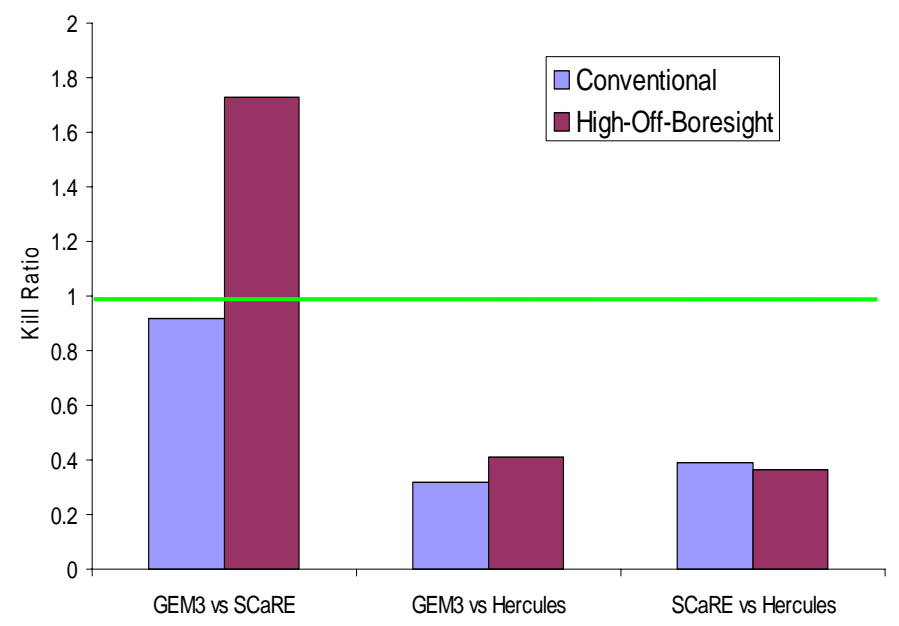

Figure 11 - Slow-speed scissor kill ratio results 
sometimes inadequate, and the criteria used by the pilots in deciding to take a shot or not therefore became somewhat subjective. Additional, more in-depth analysis was conducted in order to better understand the results.

\section{B. Engagement Analysis Results}

More detailed engagement analysis was conducted to attempt an understanding of why the raw results did not lead to unambiguous conclusions regarding the effectiveness of the different aircraft models. This analysis consisted of calculation of 'offensive advantage' and first shot opportunities. This eliminates the pilot employment, cueing, and missile flyout issues that may have impacted the exchange ratio results. It provides insight into the sometimes inconsistent top-level results by more closely examining how the different aircraft models were maneuvered and examines what 'could' or 'should' have happened in each engagement.

In examining the time of offensive advantage for the high-aspect (turning fight), conventional weapons case, it was determined that the high fidelity GEM model had a significant advantage over both the SCaRE and Hercules

\begin{tabular}{|c|c|c|c|c|}
\hline \multicolumn{5}{|c|}{ High-Aspect Engagements } \\
\hline $\begin{array}{l}\text { Pilot } \\
\text { Pair }\end{array}$ & $\begin{array}{l}\text { First } \\
\text { Pilot } \\
\text { Kills } \\
\text { Conv } \\
\text { Wpns }\end{array}$ & $\begin{array}{l}\text { Second } \\
\text { Pilot } \\
\text { Kills } \\
\text { Conv } \\
\text { Wpns }\end{array}$ & $\begin{array}{l}\text { First } \\
\text { Pilot } \\
\text { Kills } \\
\text { HOBS } \\
\text { Wpns }\end{array}$ & $\begin{array}{l}\text { Second } \\
\text { Pilot } \\
\text { Kills } \\
\text { HOBS } \\
\text { Wpns }\end{array}$ \\
\hline A vs. B & 17 & 8 & 21 & 26 \\
\hline A vs. C & 17 & 12 & 28 & 6 \\
\hline B vs. C & 8 & 16 & 22 & 11 \\
\hline \multicolumn{5}{|c|}{ Slow-Speed Engagements } \\
\hline $\begin{array}{l}\text { Pilot } \\
\text { Pair }\end{array}$ & $\begin{array}{l}\text { First } \\
\text { Pilot } \\
\text { Kills } \\
\text { Conv } \\
\text { Wpns }\end{array}$ & $\begin{array}{l}\text { Second } \\
\text { Pilot } \\
\text { Kills } \\
\text { Conv } \\
\text { Wpns }\end{array}$ & $\begin{array}{l}\text { First } \\
\text { Pilot } \\
\text { Kills } \\
\text { HOBS } \\
\text { Wpns }\end{array}$ & $\begin{array}{l}\text { Second } \\
\text { Pilot } \\
\text { Kills } \\
\text { HOBS } \\
\text { Wpns }\end{array}$ \\
\hline A vs. B & 18 & 8 & 16 & 11 \\
\hline A vs. C & 11 & 13 & 19 & 14 \\
\hline B vs. $C$ & 6 & 17 & 18 & 13 \\
\hline
\end{tabular}

Table 2 - Pilot Kill Comparisons models (Figure 12.) In this case, offensive advantage is calculated as the time for which the shooter is within a 45 degree cone of the opponent's tail. Pilot comments indicated that they felt that the nose-pointing authority of the GEM model was superior to the other two models.

When high off-boresight weapons are introduced, the results between the different aircraft are significantly closer, as seen in Figure 13. Quantitative results on high-aspect missile with HMS first-shot opportunities were calculated by defining a valid launch envelope if the target is within a cone of azimuth/elevation $+\alpha \leq 75^{\circ}$, and range $>1500$ $\mathrm{ft}$.

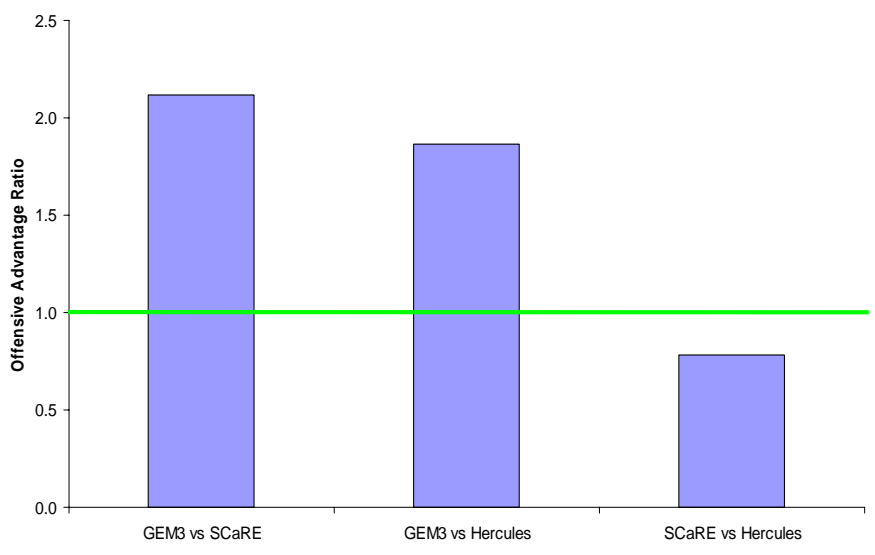

Figure 12- Ratio of time at offensive advantage, high-aspect scenario, conventional weapons

The data show that GEM3 had an advantage in reaching the high-off-boresight missile parameters before the SCaRE could get to the launch window. The other pairings were about equal, with a very slight disadvantage to the Hercules model. The angular advantage in being able to take the first shot was typically only about 2 degrees - so if both pilots pressed the fight, most of the engagements would result in mutual kills. The duration of the engagements before the first firing solution was typically less than 10 seconds.

The data for the slow-speed scissors setup was quite different. Here, slow-speed maneuverability was very important to enable the pilot to get the airplane within parameters

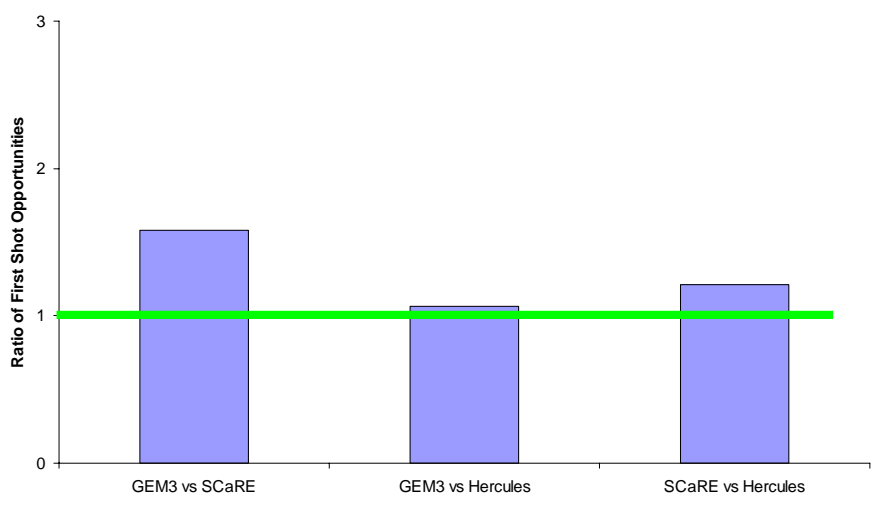

Figure 13- First shot opportunity ratios for the high-aspect setup with HMS and all-aspect missiles

American Institute of Aeronautics and Astronautics 


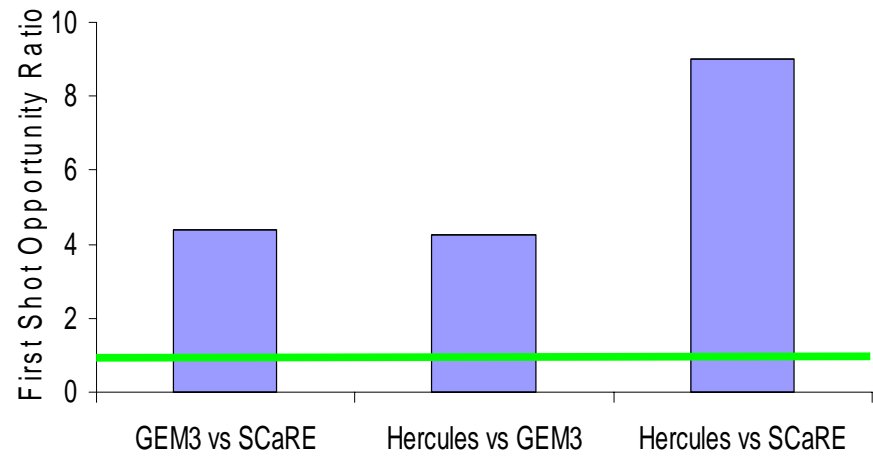

Figure 14- First missile shot opportunity ratios for the slowspeed scissors setup with HMS and all-aspect missiles

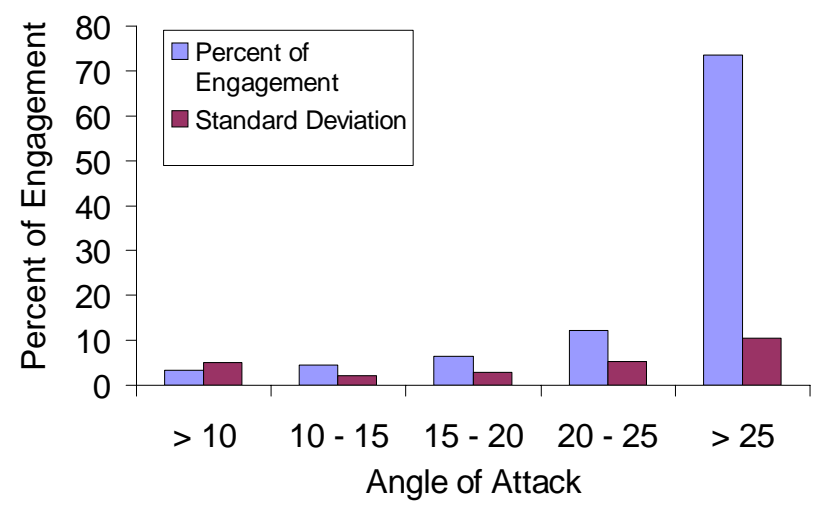

Figure 15 - Scissors engagement use of angle-of-attack

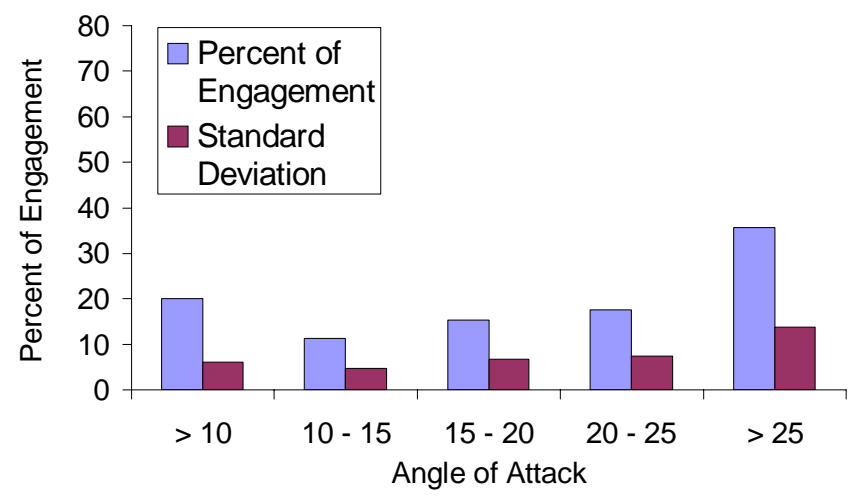

Figure 16 - High-aspect engagement use of angle-of-attack

for a missile shot. The average duration of the engagement before either airplane achieved a valid firing solution was about 40 seconds, so this indicates prolonged maneuvering at slow speeds to obtain an advantage. Figure 14 shows that the GEM3 configuration had a clear advantage over the SCaRE airplane in the slow-speed fight if he used the high-aspect missiles. Typical angular advantage of the GEM3 over the SCaRE at the time of first shot opportunity was $55^{\circ}$. The Hercules model was clearly superior in these engagements over both the GEM3 and SCaRE models (note that the presentation in figure 14 has changed to result in positive ratio information by inverting the normal presentation of the Hercules results against the higher fidelity models). The first shot opportunity ratio of Hercules to GEM3 was about 4-to- 1 with a $58^{\circ}$ advantage on the average, and Hercules vs. SCaRE was about 9to-1 with an $81^{\circ}$ advantage on the average. As noted previously, one of the key differences giving the Hercules model an advantage in the slow-speed scenario was the higher maximum lift available seen in Figure 4. Because the models were developed independently, the exact positioning of the aircraft center of gravity and the effectiveness of the trim surfaces varied. In the case of the Hercules model, no explicit moment balancing is used, and the model simply 'rides' the pre-defined $\mathrm{C}_{\text {Lmax }}$ curve.

It is instructive to examine the results of the slow-speed scissors cases to determine the cause for the pattern. Figure 15 shows the amount of time during the engagements the airplanes were at various $\alpha$ ranges. This did not matter which airplane model was used or which of the three pilots who flew the model there were very similar results across the board. This result showing the large amount of time at high- $\alpha$ is significant because, as previously mentioned, good aerodynamic model fidelity at high- $\alpha$ conditions - especially in maneuvering flight - currently requires experimental data to develop the highly nonlinear model. The only configuration with that data was the GEM model.

For the high-aspect setups, there were no significant differences in $\alpha$ between all-aspect missiles or conventional and not much difference between airplane and pilot. Figure 16 shows the time at specific angles-of-attack for the high-aspect setup; it is evident that it is weighted towards much less time at the high $\alpha$ 's. The fact that the high- $\alpha$ bin has as much as the data as it does is indicative of the times that the fights degenerated into scissors engagements. Although the pilots in the study did not know what the differences between the configurations were, they quickly noted that certain of the aircraft models performed better than others in these tasks. 


\section{CONCLUSIONS}

The previous study, documented in Reference 1, demonstrated that simulation of BVR air-to-air combat does not show significant dependence on airframe modeling fidelity. The simulation results in terms of exchange ratio, shooter energy at missile launch, and shot results demonstrated no discernable dependency upon the type of airframe model used by either the shooter or the target. The airframe model fidelity is certainly less critical to the simulation results than pilot proficiency or simulation hardware.

This study, which focuses on the impact of airframe model fidelity in highly dynamic, close-in combat scenarios, does show a dependency upon the methods used to develop the aircraft airframe model.

For high-aspect engagements which result in traditional turning fights, there are very small differences due to model fidelity at first examination. While the exchange ratios in the test showed no apparent trends, additional analysis shows a clear advantage for the high fidelity model, particularly with conventional weapons. When high offboresight weapons are introduced, there are still differences in the overall time of advantage in favor of the high fidelity model, but these differences are small enough to have minimal impact on the outcome of the engagement. A key issue here in deciding on fidelity requirements for a simulation study might be the metrics planned to use for analysis. For instance, first shot opportunity metrics would clearly show differences between the configurations, and studies using that metric alone might be misleading when using lower fidelity simulation models. Use of kill ratio data or including the missile fly-out timelines would show much more even outcomes (i.e. usually mutual kills), and therefore, perhaps the modeling fidelity issues would not become so important.

In scissors fights, differences in the modeling of high- $\alpha$ characteristics manifest themselves in major differences in the outcome of the engagements. For this particular case, this resulted in a clear advantage of both the Hercules (low-fidelity) model and the GEM (high-fidelity) model over the SCaRE (medium-fidelity) model. It should be noted that these results will probably vary as a function of the specific aircraft being represented, and how closely the modeled high- $\alpha$ characteristics represent reality.

In summary, this study indicates that use of a high fidelity model is indicated when:

1) The model will be used in tests where high- $\alpha$ performance is required to accurately represent aircraft capability - i.e., low-speed cases.

2) The model will be used in scenarios where precision tracking is required, such as GUNS engagements.

Using a low-fidelity, modified point-mass model is adequate when:

1) The expected angle-of-attack to be used is in "the heart of the envelope" - primarily BVR scenarios with limited WVR combat, when low-speed maneuvering is not anticipated

2) High off-boresight sensors and missiles are used

One interesting result from this study is that while the medium fidelity model handles more like a real-world aircraft than the simpler representation, the limited aerodynamic databases permits unrecoverable departures. This must be factored into consideration, since these tests should be eliminated from the results of any simulation using these models and may therefore result in lost productivity. The model provides the illusion of better performance, but in reality may be no better or possibly worse than the low fidelity model.

The results of this study provide a distinct benefit to the simulation and evaluation community. The guidance on level of fidelity, and therefore cost and effort required, is needed to provide realistic results when evaluating piloted simulation studies on close-in combat effectiveness. This is original work in gathering information specifically to address what benefits, if any, are gained by spending extra resources to develop higher fidelity models in the closein air combat arena. During close-in combat, significant maneuvering is required so this should be a critical area of the combat mission for tradeoffs and evaluations. This study did not address requirements for training simulators or high-gain tasks such as take-offs and landings. 


\footnotetext{
${ }^{1}$ Impact of Aircraft Flight Dynamics Modeling Technique on Weapon System Beyond-Visual-Range Combat Effectiveness; T. Ray Persing, Thomas Dube, Jeff Slutz; AIAA 2003-5689

${ }^{2}$ Airplane Flight Dynamics, Volume 1; Roskam, Jan; Roskam Aviation and Engineering Corporation, 1979

${ }^{3}$ LaSRS++ An Object-Oriented Framework for Real-Time Simulation of Aircraft; Richard Leslie et al; AIAA98-4529
} 